\title{
Dolor abdominal: causas poco frecuentes
}

\section{Abdominal Pain: Uncommon Causes}

\author{
Mariángela Paba ${ }^{1}$ Jairo Hernández Pinzón ${ }^{1} \quad$ Esteban Jeanmaire $^{1}$ Nebil Larrañaga ${ }^{1}$ Germán Espil ${ }^{1}$ \\ Shigeru Kozima ${ }^{1}$ \\ ${ }^{1}$ CEMIC, Buenos Aires, Argentina \\ Address for correspondence Jairo Hernández Pinzón, MSc, \\ Departamento de imágenes, Centro de Educación Medica e \\ Investigaciones Clínicas, Hospital Universitario Sede Saavedra, \\ Galván 4102, Capital Federal, Argentina \\ Rev Argent Radiol 2022;85(Suppl S1):S1-S10. \\ (e-mail: jahernandezpinzon@gmail.com).
}

\section{Resumen \\ Palabras Clave \\ - dolor abdominal \\ - etiología \\ - tomografía computada}

El dolor abdominal es una de las causas más frecuentes de consulta médica, existen algunas causas comunes con una aproximación diagnóstica sencilla; otras causas poco prevalentes representan un reto diagnóstico para el especialista en diagnóstico por imágenes debido a su presentación clínica inespecífica, baja sospecha diagnóstica y escasa frecuencia. Sin embargo, algunas de esas entidades infrecuentes presentan hallazgos característicos en los diferentes métodos de imágenes. En este trabajo, presentamos los hallazgos radiológicos de causas infrecuentes de dolor abdominal que han sido diagnosticadas en nuestra institución en los últimos 10 años.

Abdominal pain is one of the most frequent causes of medical consultation, there are some common causes with a simple diagnostic approach. However, other causes that are not prevalent represent a diagnostic challenge since their nonspecific clinical presentation and its low diagnostic suspicion due to its rarity, however, some of these infrequent entities present characteristic imaging findings. In this work, we present the radiological findings of infrequent causes of abdominal pain that have been diagnosed in our institution in the past 10 years.

\section{Introducción}

El dolor abdominal es una de las causas de consulta médica más frecuente, afecta a casi todas las personas por lo menos una vez en su vida, independientemente de la edad, género o entorno social. ${ }^{1}$ Muchas de las enfermedades que se manifiestan con ese síntoma por su alta frecuencia, son de una aproximación diagnóstica poco compleja. Sin embargo, un número importante de pacientes presenta una clínica inespecífica que no coincide con el diagnóstico real, por lo que en algunas oportunidades se hace indispensable el uso de imágenes diagnósticas. ${ }^{2}$

El uso de métodos como la tomografía computada (TC) y la resonancia magnética (RM) en casos de dolor abdominal ha aumentado 3 veces (del 5 al 17\%) en países desarrollados como Estados Unidos. ${ }^{3}$ Eso ha llevado, por un lado, a un incremento significativo en los costos de atención sanitaria $\mathrm{y}$, por otro lado, a una mayor capacidad de detección de enfermedades con poca frecuencia diagnóstica. received

May 26, 2018

accepted

October 28, 2018

published online

December 18, 2018
DOI https://doi.org/

10.1055/s-0038-1676496. ISSN 1852-9992.

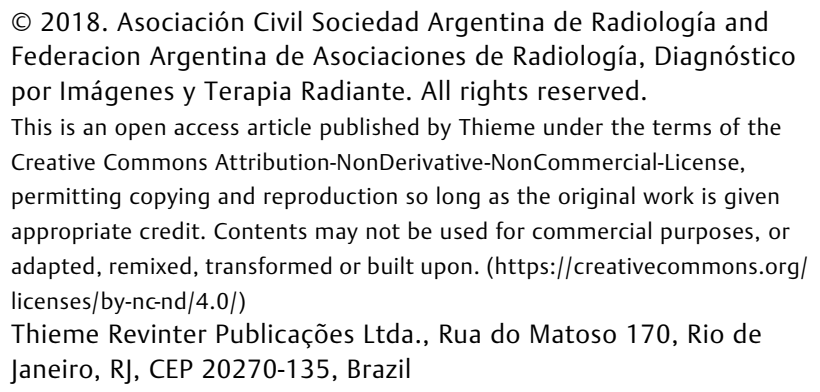


El objetivo del presente ensayo iconográfico es describir patologías poco frecuentes en la consulta por dolor abdominal, ilustrando con casos de nuestra institución.

\section{Ruptura Espontánea de Hepatocarcinoma (HCC)}

La ruptura espontánea de HCC tiene una incidencia variable, menos del $3 \%$, en países occidentales y hasta el $25 \%$ en países asiáticos. ${ }^{4}$ Es una complicación grave, en la que se reportan tasas de mortalidad de hasta el $75 \%{ }^{5}$

Existen diferentes hipótesis en cuanto a su etiología, unas hablan del rápido crecimiento y necrosis tumoral, otras de erosión de los vasos hepáticos o coagulopatía secundaria a disfunción plaquetaria. ${ }^{6}$ No obstante, ninguna de ellas ha constituido causa suficiente para explicar esta complicación. ${ }^{6}$

El ultrasonido (US) y la tomografía computada (TC) pueden mostrar la tumoración hepática asociada a hemoperitoneo. Cuando el HCC es de localización subcapsular puede verse separado y con ruptura intraperitoneal hacia el espacio perihepático, ${ }^{7}$ observándose una lesión de baja atenuación y realce periférico en la fase arterial, lo que puede asemejarse a una órbita enucleada, "signo de la enucleación". ${ }^{8}$ Otro signo reportado en TC en algunos casos es la presencia de un hematoma de alta atenuación alrededor del tumor roto, llamado signo del coágulo centinela (- Fig. 1). ${ }^{7}$

\section{Bazo Errante}

Se denomina bazo errante cuando este se desplaza de su posición normal debido a laxitud o ausencia de los ligamentos que lo mantienen en su lugar. Se calcula una incidencia del $0,2 \%$ para esta entidad, ${ }^{9}$ existiendo menos de 500 casos reportados en la literatura al momento de redacción de este ensayo. ${ }^{10}$ Esta condición puede ocasionar dolor abdominal por torsión aguda, crónica o intermitente de su pedículo vascular, pudiendo ocasionar infarto o compresión de estructuras vasculares adyacentes. ${ }^{9}$

Generalmente se produce entre los 20 y 40 años de edad y es más común en mujeres multíparas debido a la laxitud de la pared abdominal. Otra causa relacionada comúnmente es la esplenomegalia secundaria a
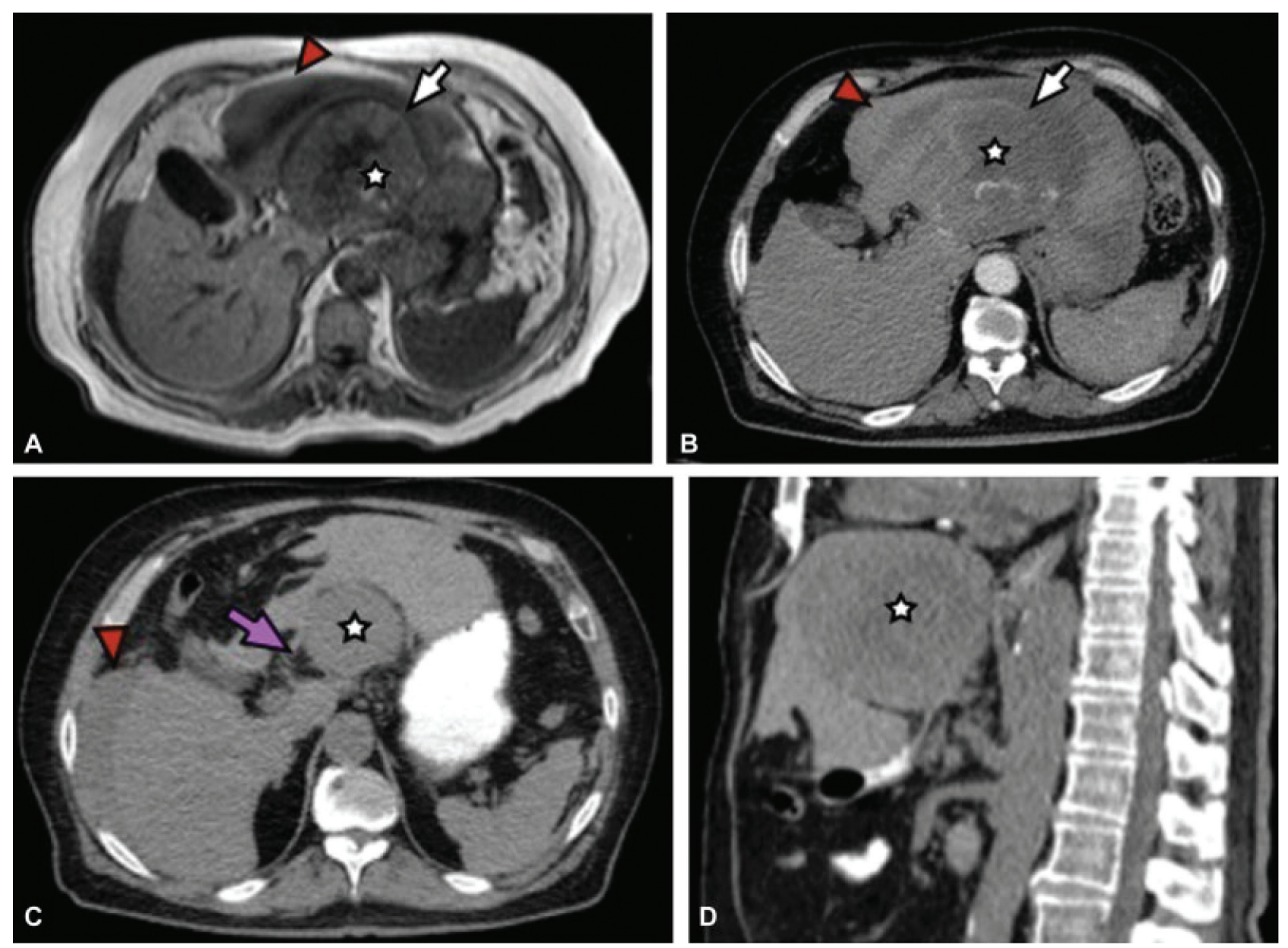

Fig. 1 Paciente masculino de 65 años de edad con antecedente de cirrosis y ruptura espontánea de hepatocarcinoma (HCC). Asistió al servicio de urgencias por dolor abdominal, por lo cual le realizaron TC y RM de abdomen. (A) Secuencia ponderada en T1, halo hipointenso (flecha blanca), liquido perihepático predominantemente hipointenso en ponderación T1 con efecto hematocrito (flecha roja). (B) TC con contraste endovenoso plano axial al mismo nivel, realce periférico alrededor del HCC (flecha blanca), líquido hiperdenso (flecha roja). (C) TC, corte axial más caudal sin contraste endovenoso, líquido hiperdenso (flecha roja), hallazgo vinculable a ruptura intraperitoneal de HCC con hemoperitoneo. (D) TC sin contraste en plano sagital, lesión nodular en lóbulo hepático izquierdo que corresponde a un HCC (estrella blanca). 

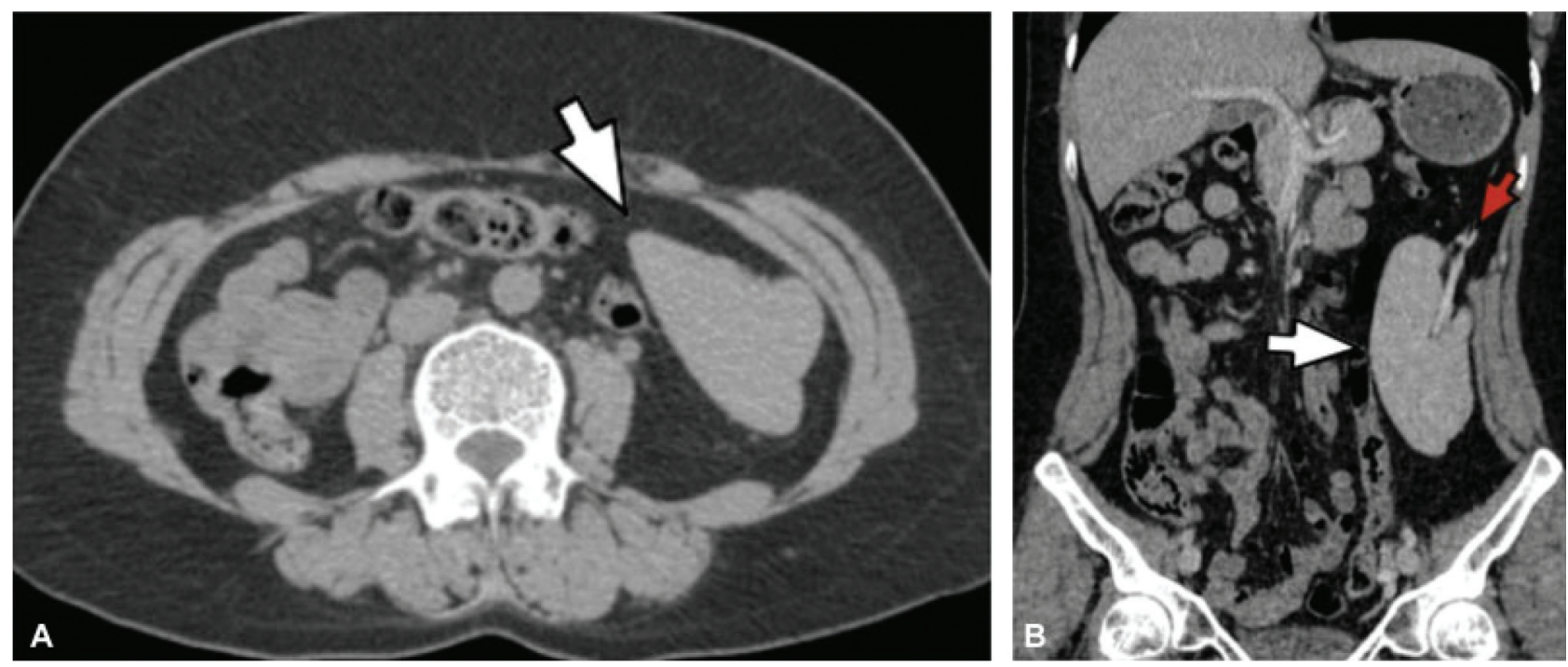

Fig. 2 Paciente femenina de 38 años de edad con dolor en hipocondrio izquierdo. (A) TC de abdomen y pelvis sin contraste en plano axial, flanco izquierdo, bazo con ubicación anómala y rotación externa de su hilio (flecha blanca). (B) TC con contraste en fase arterial plano coronal, pedículo vascular torsiónado (flecha roja) y convexidad esplénica rotada internamente.

enfermedades linfoproliferativas. ${ }^{11}$ En imágenes debemos sospecharlo cuando se observa una masa en cualquier posición abdominal con ecogenicidad en el US o realce en la TC con contraste similar al bazo, además de no observarse éste en su posición normal (-Fig. 2). ${ }^{11}$

\section{Divertículo Duodenal Roto}

La prevalencia del divertículo duodenal varía del 1 al $22 \%$ dependiendo del método de diagnóstico utilizado (por ejemplo, en TC se reporta una prevalencia del $8,3 \%)^{12}$ La perforación de esa alteración anatómica es poco común, causada en la mayoría de los casos, por procesos inflamatorios (69\%). Por su sintomatología puede ser fácilmente confundida con una colecistitis, apendicitis o úlcera duodenal perforada, por lo que son necesarias las imágenes para realizar un adecuado diagnóstico diferencial.

Los divertículos duodenales generalmente se observan en TC como una estructura con densidad de tejidos blandos que se interpone entre el duodeno y la cabeza pancreática con contenido aéreo, niveles líquido/aire, material de contraste $o$ restos alimentarios. ${ }^{13}$

Entre los hallazgos diagnósticos de esta complicación se encuentran burbujas de aire libre en el espacio peritoneal o retroperitoneal, ${ }^{14}$ así como signos radiológicos clásicos de procesos inflamatorios diverticulares (engrosamiento de la pared intestinal, liquido libre y rarefacción de la grasa adyacente) (- Fig. 3) ${ }^{13}$

\section{Infarto del Ligamento Falciforme}

Es una entidad muy poco frecuente (hasta el año 2015 existían menos de 6 casos publicados ${ }^{15}$ ). El ligamento falciforme está formado por dos capas de peritoneo y contiene una cantidad variable de grasa extraperitoneal, junto con la vena umbilical remanente y el ligamento teres. Recibe su irrigación arterial de aferentes de la vena frénica izquierda y la arteria segmentaria media del hígado y su drenaje venoso a través de la vena frénica izquierda, por lo cual es vulnerable a cualquier injuria vascular. ${ }^{16}$
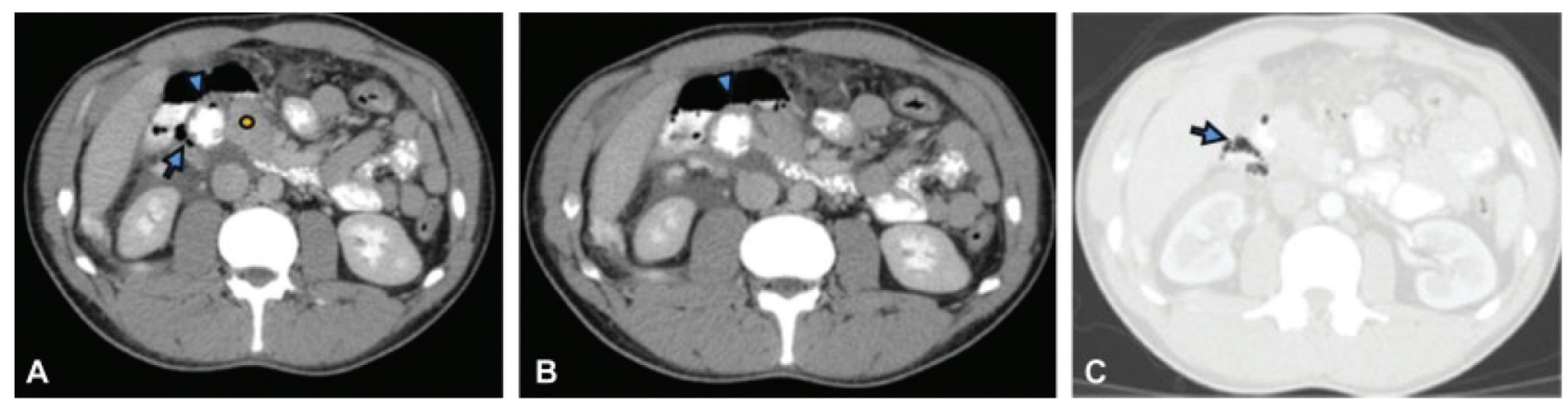

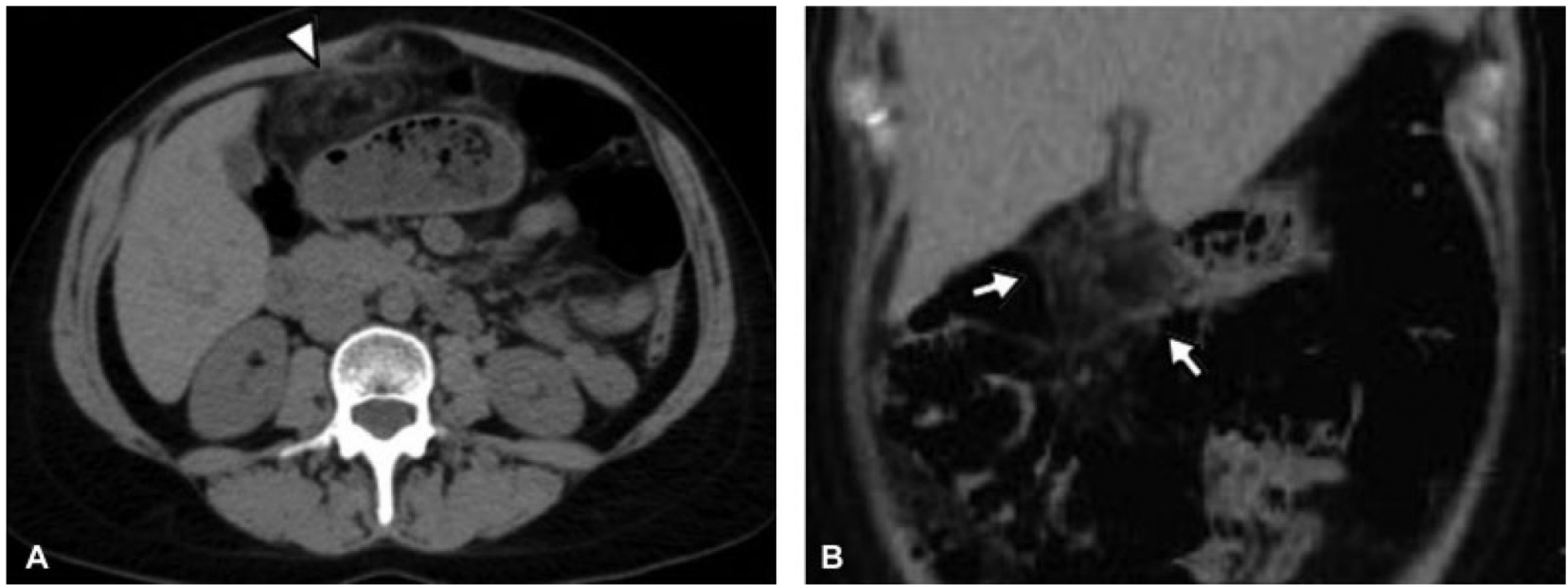

Fig. 4 Infarto del ligamento falciforme en un paciente masculino de 42 años de edad quien consultó por dolor abdominal en epigastrio al servicio de urgencias y se le realizó TC de abdomen simple; (A) y (B) rarefacción de la grasa que se encuentra en la topografía esperada del ligamento falciforme.

Esta patología tiene una presentación clínica similar al infarto omental (peritonismo, ausencia de fiebre $y$ leucocitosis), en la ecografía puede observarse como una zona hiperecogénica o heterogénea no compresible que concuerda con el máximo punto de dolor mientras que en TC se pueden observar cambios inflamatorios asociados a rarefacción de la grasa adyacente (-Fig. 4).

\section{Sindrome de Fitz Huggs Curtis}

También conocido como perihepatitis, es una rara complicación de la enfermedad pélvica inflamatoria (EPI), que se presenta en alrededor del 1 al $30 \%$ de los pacientes. ${ }^{17,18}$ Se define como una inflamación de la cápsula peritoneal que recubre el hígado y se cree que es debida a la diseminación ascendente a través de la gotera parietocólica derecha de una infección pélvica. ${ }^{17}$
Suele presentarse con dolor abdominal en el hipocondrio derecho que empeora con la inspiración y tensión de los músculos abdominales, un cuadro clínico común en múltiples patologías como colecistitis aguda, pielonefritis y dolor pleurítico (sus principales diagnósticos diferenciales). En TC se presenta con engrosamiento y realce intenso de la capsula hepática, principalmente en fase arterial (-Fig. 5).

La inflamación de la cápsula hepática puede producir áreas de alteración variable de la perfusión subcapsular o periportal, igualmente se puede observar líquido y rarefacción de la grasa en la gotiera parietocólica derecha, septos peritoneales, fluido perihepático loculado y engrosamiento de la pared vesicular. ${ }^{17-19}$ El aislamiento de Neiseria Gonorreae o Clamidia Tracomatis en el estudio del fluido vaginal sumado a las imágenes clásicas descriptas es suficiente para confirmar el diagnóstico. ${ }^{17}$
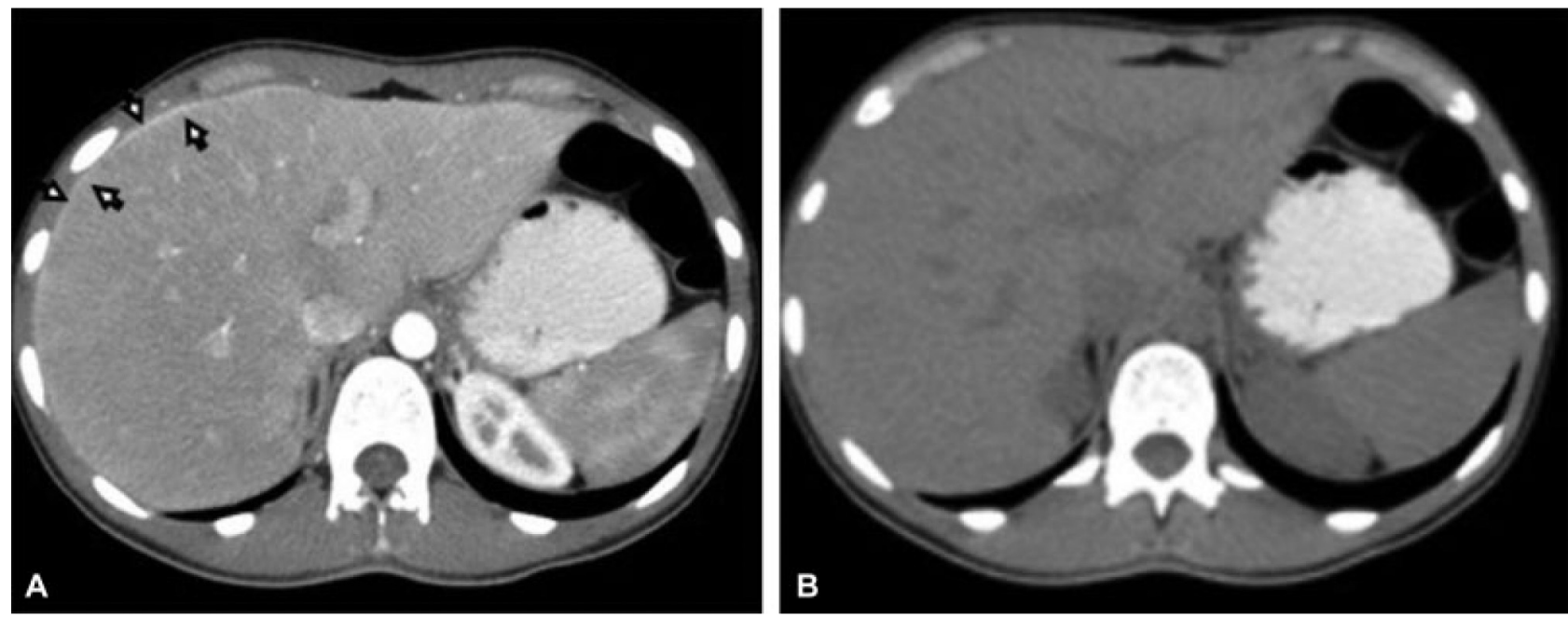

Fig. 5 Paciente de sexo femenino de 23 años de edad con antecedente de enfermedad pélvica inflamatoria a quien se le realizó TC de abdomen con contraste oral y endovenoso debido a dolor en hipocondrio derecho. En la fase arterial (A) se marca con las flechas intenso realce de la cápsula hepática, en (B) TC sin contraste al mismo nivel de corte. 

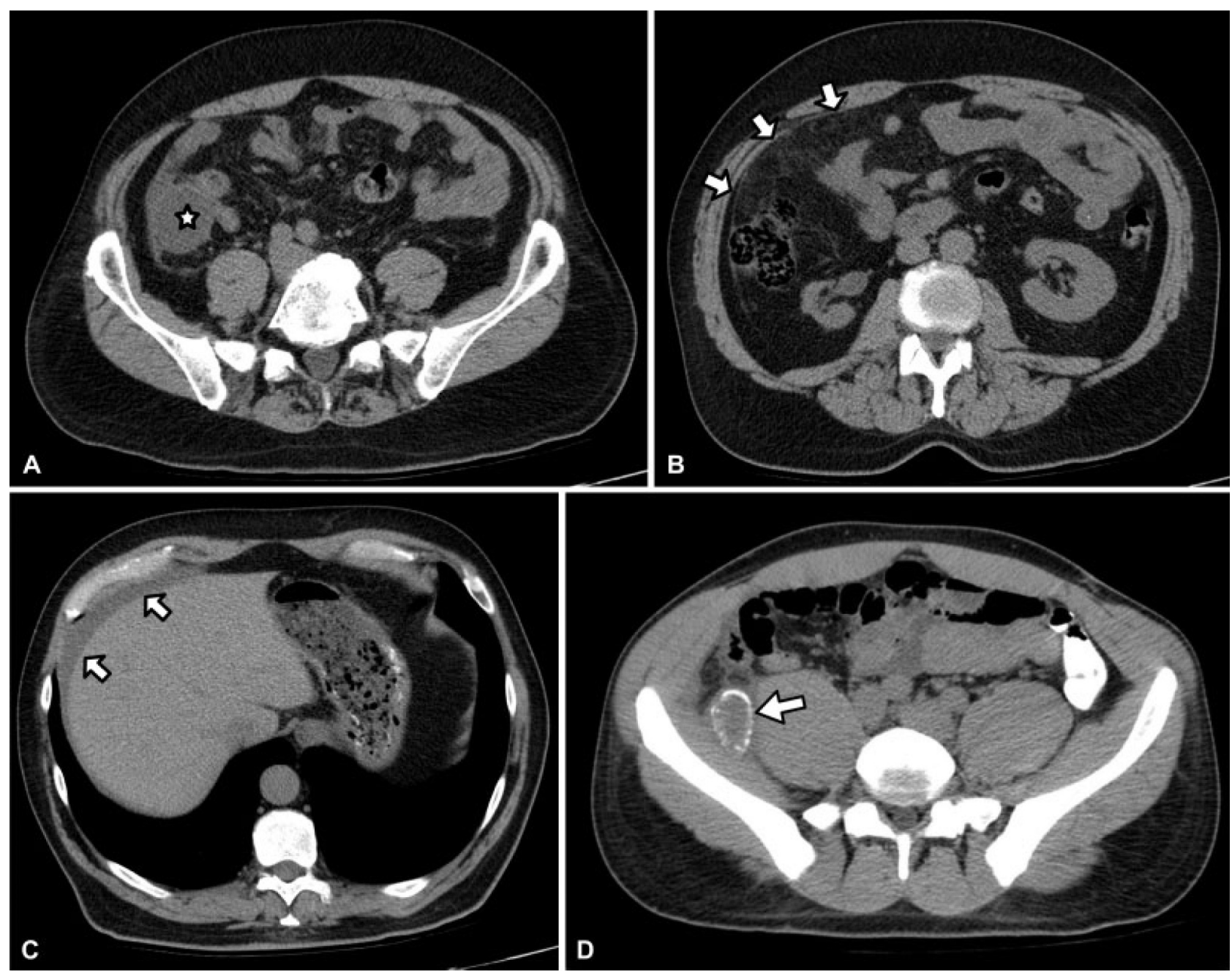

Fig. 6 Paciente masculino de 52 años de edad con clínica de abdomen agudo a quien se le realizó una TC. En (A) plano axial se observa imagen quística de baja atenuación en la topografía del apéndice cecal (estrella), en (B) una imagen más cefálica muestra rarefacción de la grasa periférica (flechas) en relación a la infiltración carcinomatosa; en (C) se señala material mucoso ubicado entre el borde hepático anterior y la pared abdominal. En la figura (D) se muestra otro paciente diferente de sexo masculino, con 23 años de edad, quien consultó por dolor agudo en fosa iliaca derecha, y en la TC de abdomen se identificó un mucocele con calcificaciones parietales (flecha).

\section{Mucocele Apendicular}

El mucocele apendicular es una acumulación de moco en la luz del apéndice cecal que produce su dilatación secundaria a obstrucción. $^{20}$ Es una entidad poco frecuente, algunos artículos reportan una prevalencia del $0,3 \%$ en todas las apendicectomías, siendo más frecuente en mujeres y en mayores de 50 años. ${ }^{20}$ La presentación clínica es variable, pudiendo presentarse como dolor abdominal inespecífico, o con síntomas similares a la apendicitis aguda. Sin embargo, cerca del $25 \%$ de los mucoceles, son asintomáticos y hallados de forma incidental. ${ }^{20}$

Los estudios por imágenes tienen un rol importante en el diagnóstico, la TC es el estudio de elección. Los hallazgos que permiten diferenciarlo de otras patologías son su aspecto quístico con forma ovalada, la baja atenuación con realce de la pared, calcificaciones parietales (hasta en el 50\% de los casos) y la ausencia de signos inflamatorios clásicos de apendicitis aguda. Sin embargo, hay que prestar atención a la infiltración del tejido adyacente que puede ser indicativa de su transformación maligna (-Fig. 6). ${ }^{21}$

\section{Angioedema Intestinal}

El angioedema intestinal es el edema submucoso de la pared intestinal, debido a la vasodilatación con acumulación de líquido en el espacio intersticial. ${ }^{22}$ Las dos causas más frecuentes son la medicamentosa principalmente por IECA (inhibidores de la enzima de conversión de la angiotensina) probablemente secundario a la degradación de bradiquininas y la hereditaria causada por la deficiencia del inhibidor de la enzima $\mathrm{C} 1 .^{23}$

Los hallazgos imagenológicos consisten en edema de la pared intestinal causado por el aumento de la permeabilidad de la vasa vasorum, engrosamiento parietal y rectificación del asa intestinal afectada la cual es principalmente un asa yeyunal. La TC muestra una baja densidad de la submucosa en comparación con la mucosa y serosa, dando el aspecto de pared estratificada que es más evidente en los estudios con 

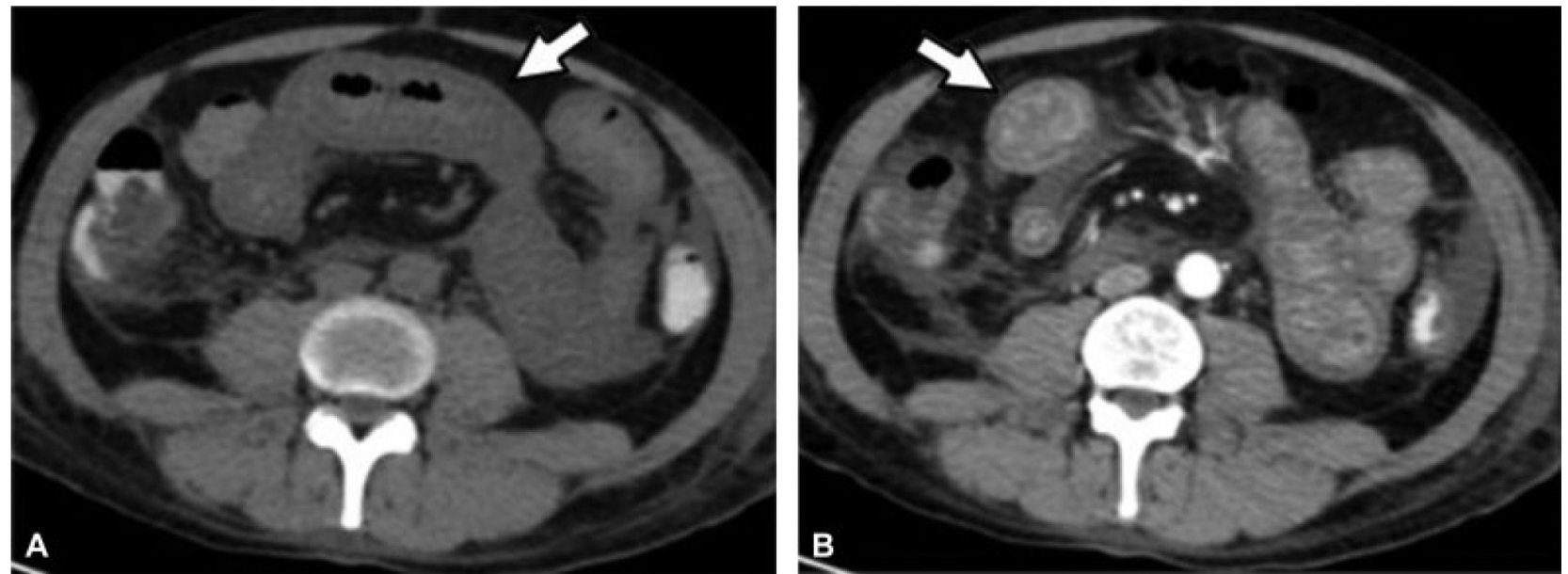

Fig. 7 Paciente masculino de 63 años de edad, medicado con inhibidores de la enzima de conversión de la angiotensina (IECA) para el tratamiento de hipertensión arterial (HTA). Asistió al servicio de urgencia por diarrea y dolor abdominal de varias semanas. Le realizaron TC; en (A) plano axial se observa edema de la pared del yeyuno proximal con rectificación del mismo (flecha). La figura (B) tras la administración de contraste endovenoso muestra patrón estratificado (flecha) asociado a líquido libre laminar y leve ingurgitación de los vasos adyacentes.

contraste endovenoso. Otros hallazgos asociados son ascitis, líquido en la luz del asa afectada y alteración de la grasa mesentérica (-Fig. 7).

\section{Diverticulitis de Meckel}

El divertículo de Meckel es la anomalía congénita más común del tracto gastrointestinal, su origen se debe a la falta de involución del conducto onfalomesentérico y su localización más frecuente es en el borde antimesentérico del íleon terminal en los primeros $100 \mathrm{~cm}$ distales a la válvula ileocecal. ${ }^{24}$

Su incidencia es de hasta el $2 \%$ en la población general, permaneciendo asintomático la mayoría del tiempo. ${ }^{24} \mathrm{La}$ frecuencia del divertículo de Meckel en hombres y mujeres tiende a ser similar, siendo más frecuentes las complicaciones en hombres. ${ }^{25}$ En orden de frecuencia son: hemorragia gastrointestinal secundaria a ulceración de tejido gástrico ectópico; obstrucción intestinal causada por invaginación, vólvulos o estenosis; diverticulitis, perforación y en última instancia transformacion neoplásica. ${ }^{26}$
La presentación clínica de la diverticulitis de Meckel muchas veces es indistinguible de una apendicitis aguda, cursando con dolor abdominal en la fosa ilíaca derecha, fiebre y vómitos. La TC desempeña un papel vital para su diagnóstico, pudiendo demostrar una imagen redondeada o tubular de tamaño variable entre 2-6 cm en comunicación con el intestino delgado ( - Fig. 8), la cual puede realzar tras la administración de contraste endovenoso y presenta engrosamiento parietal asociado a rarefacción de la grasa adyacente. ${ }^{27}$

\section{Sindrome de Wunderlich}

El síndrome de Wunderlich es el sangrado renal espontáneo confinado al espacio perinéfrico y subcapsular de causa no traumática, ${ }^{28}$ es infrecuente y presenta una incidencia similar entre hombres (55\%) y mujeres (45\%). ${ }^{28}$

La etiología es variable con causas benignas y malignas; cerca del $50 \%$ son secundarios a tumores, siendo el carcinoma de células renales y el angiomiolipoma las patologías involucradas con mayor frecuencia; otras causas menos
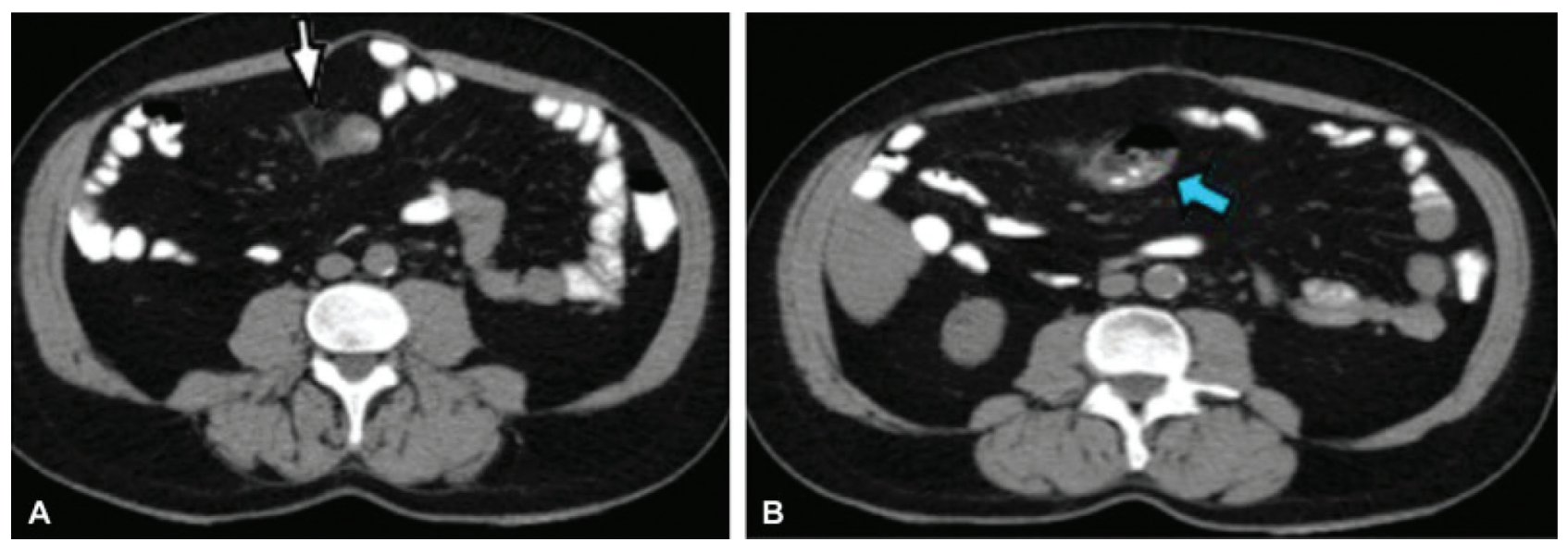

Fig. 8 . En (A) TC plano axial donde se muestra un divertículo de Meckel (flecha) con rarefacción de la grasa adyacente. La figura (B) muestra un corte más cefálico, imagen con densidad cálcica al interior del divertículo compatible con enterolito. 

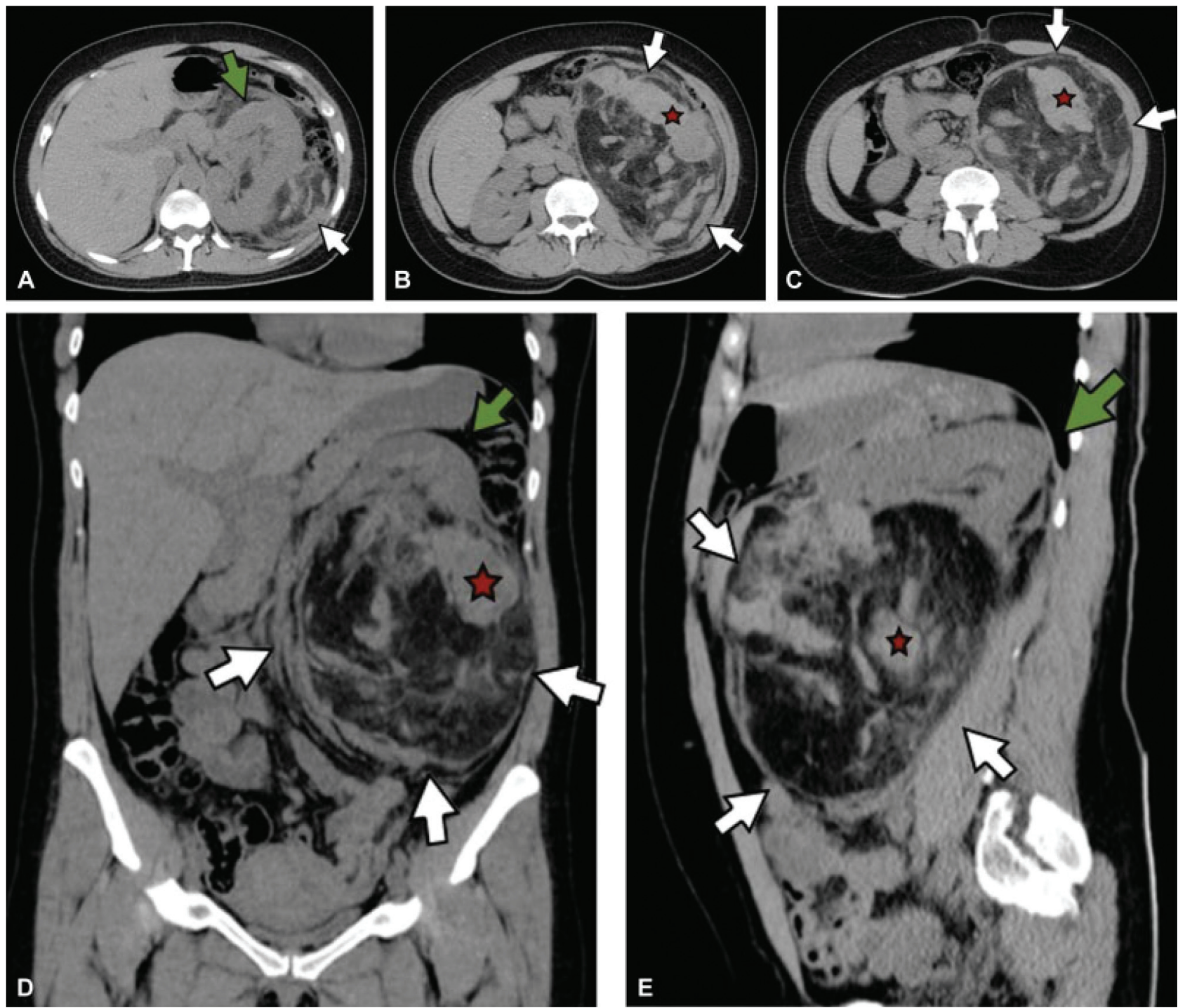

Fig. 9. Paciente masculino de 53 años de edad con antecedente de angiomiolipoma, que asistió al servicio de urgencias por dolor abdominal agudo asociado a formación de masa a nivel del flanco izquierdo. Le realizaron TC, en donde se observó el riñón izquierdo aumentado de tamaño (flecha verde en a, d y e) y desplazado cefálicamente, en su polo inferior, se observa el angiomiolipoma (flechas blancas en a-e) con áreas espontáneamente hiperdensas vinculables a sangrado agudo (estrellas rojas en b-e).
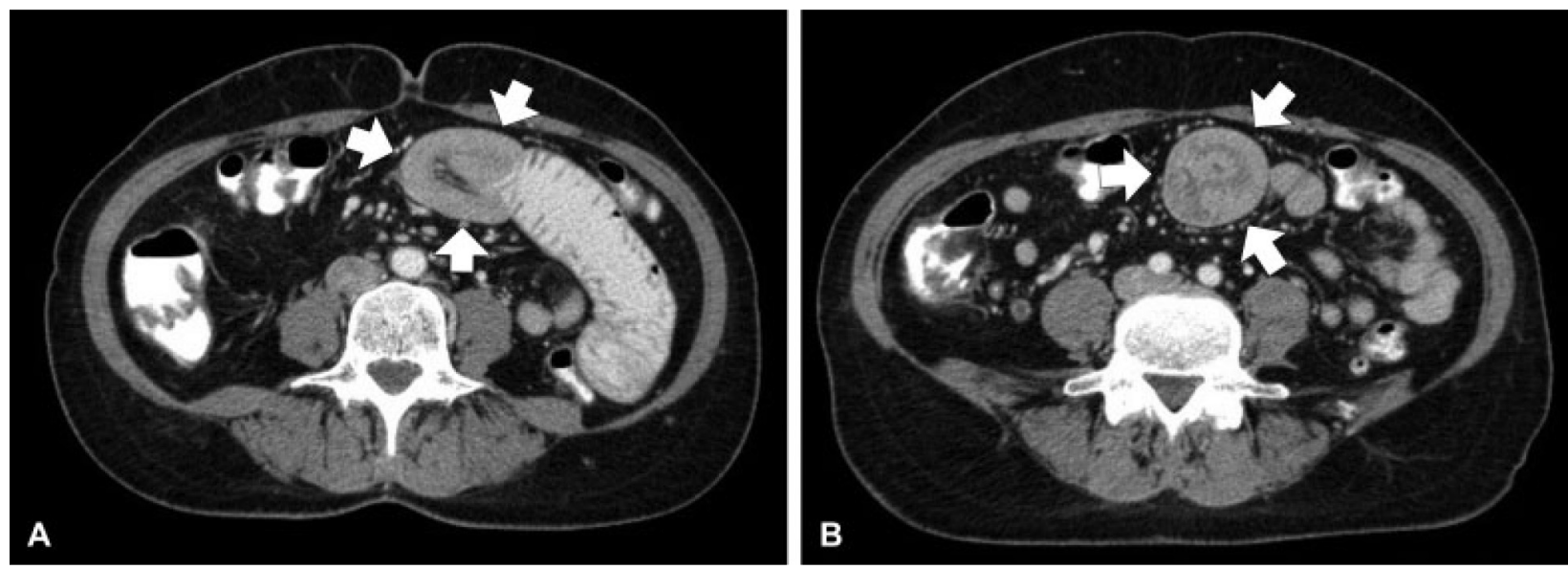

Fig. 10 Paciente femenina de 39 años de edad con antecedente de melanoma cutáneo quien asistió al servicio de urgencias por clínica de oclusión intestinal. Se realizó TC de abdomen con contraste oral y endovenoso, en (A) se señala un asa invagina al interior del asa invaginante conformando el signo de la "salchicha" asociado a dilatación intestinal retrógrada; en un corte más distal (B) se señala la invaginación conformando el signo en diana a nivel del yeyuno. 

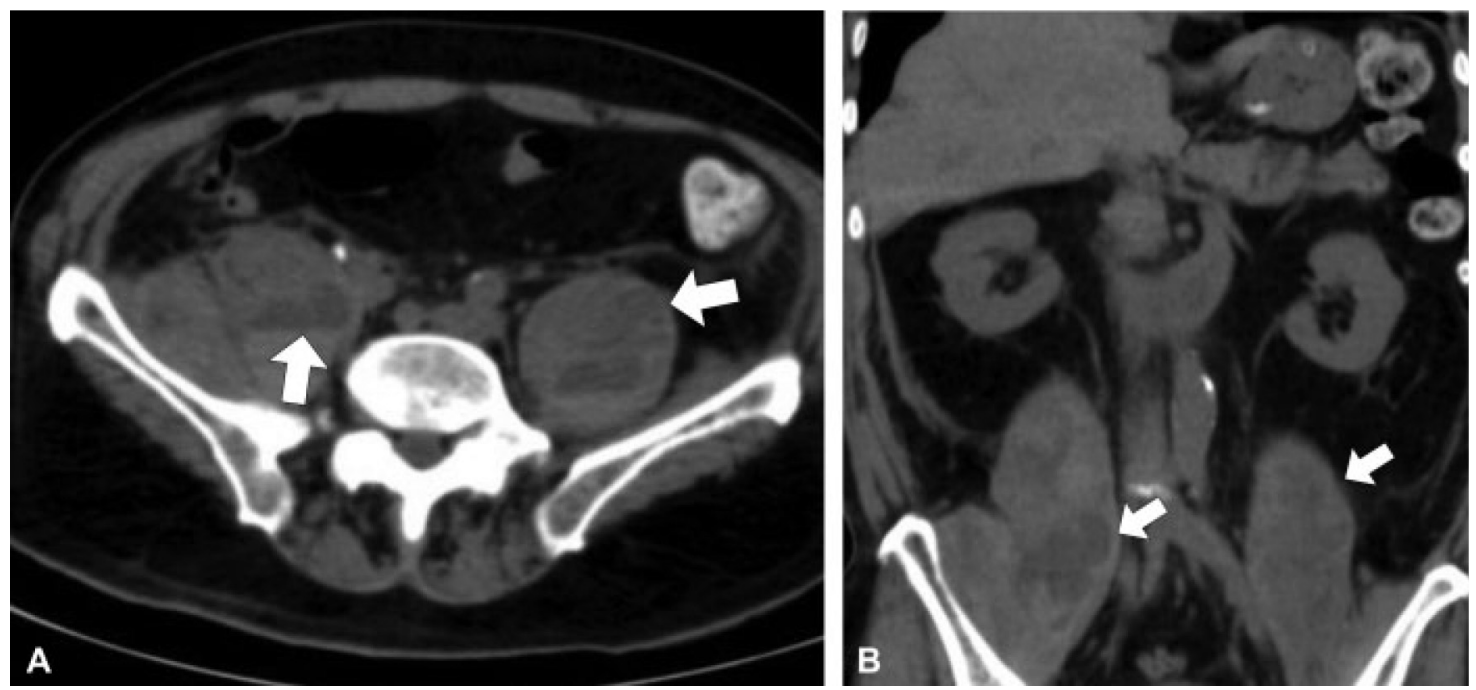

Fig. 11 Paciente con abscesos en ambos músculos psoas. En (A) se muestra TC de abdomen plano axial y en (B) plano coronal donde se señalan áreas hipodensas (flechas) a nivel a ambos músculos psoas.

frecuentes son las malformaciones arteriovenosas, quistes renales o trastornos de la coagulación. ${ }^{29}$

Clínicamente es característico el dolor abdominal súbito referido al flanco, la formación rápida de una masa palpable y signos de shock hipovolémico (cuadro clínico denominado como la triada de Lenk). ${ }^{29}$

La ecografía es en muchas ocasiones el método inicial de abordaje y se puede observar un hematoma perinéfrico el cual comprime y desplaza el parénquima renal. ${ }^{30}$ La TC (método de elección) muestra una colección hemática perirrenal o subcapsular con efecto de masa y en las series posteriores a la administración de contraste se pueden ver signos de sangrado activo (-Fig. 9).

La RM y arteriografía pueden ser útiles en aquellos casos en que la causa del sangrado no es identificada por medio de la TC. ${ }^{30}$

\section{Invaginación Intestinal por Metástasis de Melanoma}

En adultos se considera una rareza, siendo causas de oclusión intestinal solo hasta en el $3 \%$ de algunas series. ${ }^{31}$ Clínicamente se presenta como una obstrucción mecánica, parcial o completa asociada a dolor tipo cólico. Otros síntomas pueden ser vómitos, sangrado y estreñimiento. ${ }^{32}$

El melanoma maligno superficial es la forma cutánea más frecuente y por lo tanto la que con mayor frecuencia se implica en metástasis al intestino delgado, afecta por igual al yeyuno e íleon. ${ }^{33,34}$ Existen cuatro tipos de presentación del melanoma metastásico: en intestino delgado, cavitario, infiltrante, exo-entérico y polipoideo, ${ }^{34}$ siendo el último la presentación más propensa a producir invaginaciones. ${ }^{35}$

En la TC se pueden observar tres patrones diferentes dependiendo del plano de sección visualizado y la severidad del cuadro, la imagen en "diana" aparece en la fase precoz en el corte transversal, imagen en forma de "salchicha" en el corte longitudinal o "pseudo-riñón” en la fase más severa debido al edema y compromiso vascular ( - Fig. 10). ${ }^{36}$

\section{Absceso del Psoas}

El absceso de psoas se clasifica en primario y secundario. ${ }^{37} \mathrm{El}$ primario es infrecuente y usualmente idiopático (61\% de los casos), los pacientes inmunocomprometidos son los más propensos, en especial los que reciben tratamiento inmunosupresor. Generalmente se desarrolla a partir de la propagación de enfermedades intestinales (apendicitis o diverticulitis), renales (pielonefritis) u óseas (osetomielitis). ${ }^{38}$ El organismo más frecuentemente aislado es el stafilococcus aureus (88\% de los casos) seguido por la Escherichia coli y el streptococcus. ${ }^{39}$ El riesgo de mortalidad en pacientes no tratados puede llegar al $100 \%,{ }^{40}$ por lo que es importante hacer un diagnóstico temprano. Sin embargo, los síntomas son inespecíficos, se ha descripto una triada clásica de fiebre, dolor de espalda y espasmos del psoas en el $30 \%$ de los pacientes. ${ }^{41}$

La TC con contraste es el método de elección para el diagnóstico. ${ }^{38,42}$ Las imágenes muestran una lesión hipodensa con realce en anillo en el músculo psoas ( $\mathbf{F i g . ~ 1 1 ) . ~}{ }^{43}$

\section{Conclusión}

El dolor abdominal es uno de los síntomas de consulta más frecuente, tanto en el ámbito ambulatorio como en los servicios de urgencias. Es importante que los especialistas en diagnóstico por imágenes conozcan algunas de las causas poco frecuentes de dolor abdominal y su presentación imagenológica, ya que de una adecuada aproximación diagnostica depende un adecuado tratamiento.

\section{Responsabilidades Éticas}

Protección de personas y animales. Los autores declaran que para esta investigación no se han realizado experimentos en seres humanos ni en animales.

Confidencialidad de los datos. Los autores declaran que han seguido los protocolos de su centro de trabajo sobre la publicación de datos de pacientes. 
Derecho a la privacidad y consentimiento informado. Los autores declaran que en este artículo no aparecen datos de pacientes.

\section{Confidencialidad de Los Datos}

Los autores declaran que han seguido los protocolos de su centro de trabajo sobre la publicación de datos de pacientes y que todos los pacientes incluidos en el estudio han recibido información suficiente y han dado su consentimiento informado por escrito.

\section{Conflicto de Intereses}

Los autores declaran no tener ningún conflicto de interés, con excepción del Dr. Kozima que declara como posible conflicto de interés ser miembro de la comisión directiva de la SAR y de los Dres. Hernández Pinzón y Espil que declaran como posible conflicto de interés ser revisores de la RAR.

\section{Bibliografía}

1 Viniol A, Keunecke C, Biroga T, Stadje R, Dornieden K, Bösner S, et al. Studies of the symptom abdominal pain-a systematic review and meta-analysis. Fam Pract 2014;31(05):517-529

2 Eisenberg JD, Reisner AT, Binder WD, Zaheer A, Gunn ML, Linnau $\mathrm{KF}$, et al. Role of CT in the Diagnosis of Nonspecific Abdominal Pain: A Multicenter Analysis. AJR Am J Roentgenol 2017;208(03): 570-576

3 Matz K, Britt T, LaBond V. CT ordering patterns for abdominal pain by physician in triage. Am J Emerg Med 2017;35(07):974-977

4 Zhong F, Cheng XS, He K, Sun SB, Zhou J, Chen HM. Treatment outcomes of spontaneous rupture of hepatocellular carcinoma with hemorrhagic shock: a multicenter study. Springerplus 2016; 5(01):1101

5 Lai ECH, Lau WY. Spontaneous rupture of hepatocellular carcinoma: a systematic review. Arch Surg 2006;141(02):191-198

6 Zhu LX, Liu Y, Fan ST. Ultrastructural study of the vascular endothelium of patients with spontaneous rupture of hepatocellular carcinoma. Asian J Surg 2002;25(02):157-162

7 Choi BG, Park SH, Byun JY, Jung SE, Choi KH, Han JY. The findings of ruptured hepatocellular carcinoma on helical CT. Br J Radiol 2001; 74(878):142-146

8 Singhal M, Sinha U, Kalra N, Duseja A, Khandelwal N. Enucleation Sign: A Computed Tomographic Appearance of Ruptured Hepatocellular Carcinoma. J Clin Exp Hepatol 2016;6(04):335-336

9 Wallace S, Herer E, Kiraly J, Valikangas E, Rahmani R. A wandering spleen: unusual cause of a pelvic mass. Obstet Gynecol 2008;112 (2 Pt 2):478-480

10 Irpatgire R, Kale D. The wandering spleen torsion. Int J Med Res Rev 2015;3(10):1276-1278

11 Yildiz AE, Ariyurek MO, Karcaaltincaba M. Splenic anomalies of shape, size, and location: pictorial essay. ScientificWorldJournal 2013;2013:321810

12 Rekha BM, Chandramohan A, Chandran BS, Jayaseelan V, Suganthy J. Contrast Enhanced Computed Tomographic Study on the Prevalence of Duodenal Diverticulum in Indian Population. J Clin Diagn Res 2016;10(04):AC12-AC15

13 Rao PM. Diagnosis please. Case 11: perforated duodenal diverticulitis. Radiology 1999;211(03):711-713

14 Koh YX, Chok AY, Wong ASY. Perforated duodenal diverticulum as an unusual sequelae of intestinal obstruction. ANZ J Surg 2016;86 (06):516-517

15 Maccallum C, Eaton S, Chubb D, Franzi S. Torsion of Fatty Appendage of Falciform Ligament: Acute Abdomen in a Child. Case Rep Radiol 2015;2015:293491
16 Lloyd T. Primary torsion of the falciform ligament: computed tomography and ultrasound findings. Australas Radiol 2006;50 (03):252-254

17 Revzin MV, Mathur M, Dave HB, Macer ML, Spektor M. Pelvic Inflammatory Disease: Multimodality Imaging Approach with Clinical-Pathologic Correlation. Radiographics 2016;36(05): 1579-1596

18 Simon EM, April MD. Fitz-Hugh-Curtis Syndrome. J Emerg Med 2016;50(04):e197-e198

19 Wang PY, Zhang L, Wang X, Li X-J, Chen L, Wang X, et al. Fitz-HughCurtis syndrome: clinical diagnostic value of dynamic enhanced MSCT. J Phys Ther Sci 2015;27(06):1641-1644

20 Wang $\mathrm{H}$, Chen YQ, Wei R, Wang Q-B, Song B, Wang C-Y, et al. Appendiceal mucocele: A diagnostic dilemma in differentiating malignant from benign lesions with CT. AJR Am J Roentgenol 2013;201(04):W590-5

21 Pickhardt PJ, Levy AD, Rohrmann CA Jr, Kende AI. Primary neoplasms of the appendix: radiologic spectrum of disease with pathologic correlation. Radiographics 2003;23(03): 645-662

22 Scheirey CD, Scholz FJ, Shortsleeve MJ, Katz DS. Angiotensinconverting enzyme inhibitor-induced small-bowel angioedema: clinical and imaging findings in 20 patients. AJR Am J Roentgenol 2011;197(02):393-398

23 Vallurupalli K, Coakley KJ. MDCT features of angiotensinconverting enzyme inhibitor-induced visceral angioedema. AJR Am J Roentgenol 2011;196(04):W405-11

24 Park JJ, Wolff BG, Tollefson MK, Walsh EE, Larson DR. Meckel diverticulum: the Mayo Clinic experience with 1476 patients (1950-2002). Ann Surg 2005;241(03):529-533

25 Sagar J, Kumar V, Shah DK. Meckel's diverticulum: a systematic review. J R Soc Med 2006;99(10):501-505

26 Burjonrappa S, Khaing P. Meckel's diverticulum and ectopic epithelium: Evaluation of a complex relationship. J Indian Assoc Pediatr Surg 2014;19(02):85-89

27 Kawamoto S, Raman SP, Blackford A, Hruban RH, Fishman EK. CT Detection of Symptomatic and Asymptomatic Meckel Diverticulum. AJR Am J Roentgenol 2015;205:281-291

28 Estrada J, Duarte C. Wunderlich syndrome: an unusual cause of acute abdominal pain. Urol Colomb 2010;19(02):53-57

29 Collera P, Sales R, Villa V, Caballé J, Mas A, Valencoso O, et al. Síndrome de Wünderlich. Hemorragia renal espontánea. Cir Esp 2000;68:493-519

30 Katabathina VS, Katre R, Prasad SR, Surabhi VR, Shanbhogue AK, Sunnapwar A. Wunderlich syndrome: cross-sectional imaging review. J Comput Assist Tomogr 2011;35(04):425-433

31 Resta G, Anania G, Messina F, Tullio D, Ferrocci G, Zanzi F, et al. Jejuno-jejunal invagination due to intestinal melanoma. World J Gastroenterol 2007;13(02):310-312

32 Gayer G, Zissin R, Apter S, Papa M, Hertz M. Pictorial review: adult intussusception-a CT diagnosis. Br J Radiol 2002;75(890): 185-190

33 Lens M, Bataille V, Krivokapic Z. Melanoma of the small intestine. Lancet Oncol 2009;10(05):516-521

34 Huang YJ, Wu MH, Lin MT. Multiple small-bowel intussusceptions caused by metastatic malignant melanoma. Am J Surg 2008;196 (03):e1-e2

35 Palau T, Alejo M, Romaguera A, de Castro X, de Caralt E, Serra C. [Small bowel intussusception caused by metastatic cutaneous melanoma]. Rev Esp Enferm Dig 2005;97(05):381-383

36 Boudiaf M, Soyer P, Terem C, Pelage JP, Maissiat E, Rymer R. Ct evaluation of small bowel obstruction. Radiographics 2001;21 (03):613-624

37 Johannsen EC, Sifri CD, Madoff LC. Pyogenic liver abscesses. Infect Dis Clin North Am 2000;14(03):547-563, vii

38 Pearl R, Pancu D, Legome E. Hepatic abscess. J Emerg Med 2005;28 (03):337-339 
39 Lee TY, Wan YL, Tsai CC. Gas-containing liver abscess: radiological findings and clinical significance. Abdom Imaging 1994;19(01): 47-52

40 Bächler P, Baladron MJ, Menias C, Beddings I, Loch R, Zalaquett E, et al. Multimodality Imaging of Liver Infections: Differential Diagnosis and Potential Pitfalls. Radiographics 2016;36(04): 1001-1023
41 Kawoosa NU, Bashir A, Rashid B. Spontaneous cutaneous rupture of a pyogenic liver abscess. Indian J Surg 2010;72(04):339-342

42 Yang DM, Kim HN, Kang JH, Seo TS, Park CH, Kim HS. Complications of pyogenic hepatic abscess: computed tomography and clinical features. J Comput Assist Tomogr 2004;28(03):311-317

43 Mavilia MG, Molina M, Wu GY. The Evolving Nature of Hepatic Abscess: A Review. J Clin Transl Hepatol 2016;4(02):158-168 